\title{
On description of mode amplitude fluctuations in the ocean
}

\author{
A.L. VIROVLYANSKY \\ Institute of Applied Physics of Russian Academy of Sciences, 46 Ulyanov Street, 603600 Nizhny Novgorod, \\ Russia
}

\begin{abstract}
Results of Tindle and Guthrie, Felsen and other authors on studying the relationship between ray and mode representations of a field in a range-independent waveguide are used to describe wave scattering in a waveguide with large-scaled random inhomogeneities of refractive index. The notion of Fresnel zones for modes is introduced, which is analogous to the usual Fresnel zones introduced for rays. Using the Fresnel zones for modes one can simplify the analysis of mode scattering at large-scaled inhomogeneities in waveguides. Simple formulae to calculate fluctuations of mode amplitudes are obtained. They are quite similar to the well known formulae of geometrical optics and those of the Rytov method. Unlike their ray prototypes, the formulae presented here allow one to describe field fluctuations not only at the waveguide regular points but at the caustics as well.
\end{abstract}

\section{INTRODUCTION}

Field fluctuations in a medium with large-scale random inhomogeneities are often described using the well-known formula according to which the influence of inhomogeneities are taken into account by replacing the unperturbed ray complex amplitude $Q_{0}$ by

$$
Q=Q_{0} \exp \left(i k \int \delta n d s\right)
$$

Here $k$ is the wavenumber, $\delta n$ describes fluctuations of the refractive index and integral runs over an unperturbed ray trajectory. This simple and statistical averaging-suited relation is widely used to describe field fluctuations in different random media, including the ocean [1].

In this report it is shown that there is a complete analogue of this relation for modes. Moreover, there is a mode analogue for a generalizing (1) formula obtained in the framework of the Rytov method [1] to take into account the effects of diffraction. Using the relations presented here, the mode amplitude variations due to medium inhomogeneities can be approximately calculated with the same ease as variations of complex ray amplitudes within the frameworks of geometrical optics or of the Rytov method. These relations provide a deeper understanding of interconnection between mode and ray representations in a waveguide with large-scaled inhomogeneities.

\section{APPROXIMATE SOLUTION OF THE HELMHOLTZ EQUATION}

For simplicity we restrict our consideration to a two-dimentional problem. Let us introduce Cartesian coordinates $(x, z)$ in a multimode waveguide such that $z$-axis will coincide with the direction 
of the unperturbed refractive index $n$ variation. The field is excited by a point monochromatic source located at a point $\left(0, z_{0}\right)$. It is assumed to be governed by the Helmholtz equation

$$
\frac{\partial^{2} p}{\partial x^{2}}+\frac{\partial^{2} p}{\partial z^{2}}+k^{2}\left(n^{2}(z)+2 n(z) \delta n(x, z)\right) p=-2 \delta\left(x-x_{0}\right) \delta(z)
$$

where $\delta n(x, z)$ is a small fluctuating component of refractive index. Medium inhomogeneities will be assumed to be large scale, i.e. that their characteristic scales are significantly more than the wavelength.

Let us seek the solution of (2) as an expansion

$$
p(x, z)=\sum_{m} A_{m}(x) \varphi_{m}(z) e^{i k_{m} x}
$$

where $\varphi_{m}(z)$ and $k_{m}$ are, respectively, eigenfunctions and eigennumbers of unperturbed boundary value problem.

Substituting (3) into (2), we obtain the equations governing the evolution of mode amplitudes $A_{m}(x)$ along the acoustic path. These equations have the following initial conditions:

$$
A_{m}(0)=\frac{i}{k_{m}} \varphi_{m}\left(z_{0}\right)
$$

where the right-hand side is the mth mode amplitude in the unperturbed waveguide. The equations for mode amplitudes can be simplified using the WKB approximation. To this approximation the function $\varphi_{m}(z)$ between the turning points can be expressed as

$$
\varphi_{m}^{ \pm}(z)=\varphi_{m}^{+}(z)+\varphi_{m}^{-}(z)
$$

where

$$
\varphi_{m}^{ \pm}(z)=B_{m} \exp \left( \pm i \int_{z}^{z_{m a x}} \sqrt{k^{2} n^{2}(z)-k_{m}^{2}} d z \mp i \frac{\pi}{4}\right)
$$

$z_{\max }$ is the upper turning point, $B_{m}$ is the amplitude factor. Functions $\varphi_{m}^{ \pm} \exp \left(i k_{m} x\right)$ describe Brillouin waves forming an $m$ th mode. Using (5), we shall seek each mode amplitude as a sum

$$
A_{m}(x)=e^{i X_{m}^{+}(x)} \varphi_{m}^{+}\left(z_{0}\right)+e^{i X_{m}^{-}(x)} \varphi_{m}^{-}\left(z_{0}\right)
$$

Initial conditions (4) take the form $X_{m}^{+}(0)=X_{m}^{-}(0)=0$.

In [2] it is shown that the mth mode amplitude variations are mainly caused by inhomogeneities located in the neighbourhood of two geometric-optical rays (we call them mode rays) $z=z_{m}^{+}(x)$ and $z=z_{m}^{-}(x)$ originating from the source $\left(0, z_{0}\right)$ in half-planes $z>z_{0}\left({ }^{\prime}+\right.$ 'sign) and $z<z_{0}$ ('-' sign), respectively, and having the turning points at the same horizons as the given mode. This fact is clear from a physical viewpoint. In the unperturbed waveguide each mode adds in phase (constructively interferes) with the adjacent modes only along the trajectories of its mode rays [3]. Mode amplitude variations due to scattering at large-scale refractive index inhomogeneities are caused by interactions between adjacent modes. The Brilloin waves of adjacent modes will interact effectively when scattered at inhomogeneities (leading to marked change of mode amplitudes) only in locations where they add in phase, i.e. in the neighbourhood of mode rays.

In [2] it is shown that the following approximate formulae can be used to calculate mode amplitude variations:

$$
X_{m}^{ \pm}(x)=k \int d x \int d z \delta n(x, z) \Delta_{m}^{ \pm}(x, z)
$$

where

$$
\Delta_{m}^{ \pm}(x, z)=\frac{1}{\rho_{m}^{ \pm}(x)} \exp \left(i \pi \frac{\left(z-z_{m}^{ \pm}\right)^{2}}{\left(\rho_{m}^{ \pm}(x)\right)^{2}}\right)
$$




$$
\rho_{m}^{ \pm}(x)=D_{m}\left|\frac{\partial z_{m}^{ \pm}(x)}{\partial x} \tan \theta_{m}^{ \pm}(x)\right|^{1 / 2},
$$

where $D_{m}$ is the cycle length (it is the same for both trajectories $z_{m}^{ \pm}(x)$ ), $\theta_{m}^{ \pm}$are the angles of the ray trajectories inclinations with respect to the $x$-axis.

\section{ANALOGUES OF THE RYTOV METHOD AND THE METHOD OF GEOMETRICAL OPTICS FOR MODES}

The relation (7) is a complete analogue of the formula describing ray amplitude fluctuations obtained in the framework of the Rytov method [1]. The quantity $\rho_{m}$ can be considered as a radii of the first Fresnel zone. The given formula allows us to introduce the concept of Fresnel zones for modes. In the case when $\rho_{m}^{ \pm}(x)<<L$, where $L$ is the characteristic scale of inhomogeneities of refractive index, functions $\Delta_{m}^{ \pm}(x, z)$ in (6) can be approximately replaced by $\delta\left(z-z_{m}^{ \pm}(x)\right)$. Then taking into account the influence of refractive index inhomogeneities reduces to replacement of the unperturbed mode amplitude $A_{m}(0)$ by

$$
A_{m}(x)=\varphi_{m}^{+}\left(z_{0}\right) \exp \left(i k \int_{z_{m}^{+}} \delta n d s\right)+\varphi_{m}^{-}\left(z_{0}\right) \exp \left(i k \int_{z_{m}^{-}} \delta n d s\right)
$$

Integrals run over the ray trajectories $z_{m}^{ \pm}(x)$. This relation is a complete analogue of formula (1) for modes.

It can be shown that constraints used when deducing (6) and (7) are practically equivalent to standard applicability conditions in the Rytov method and in the method of geometrical optics. At the same time the relations (6) and (7), as distinct from their ray prototypes, can be used to describe wavefields not only at the regular waveguide points but at caustics as well.

Substitution (6) or (7) into (3) yields the mode representation of the wavefield in a waveguide with large-scale inhomogeneities. It can be transformed into the ray representation using the Poisson summation formula (in complete analogy with the well-known procedure, which used for a rangeindependent waveguide[3]).

\section{CONCLUSION}

The results presented here show that in each waveguide cross-section (not too large distances are implied) for each mode there are exist "sensitive spots". When inhomogeneities are placed in these spots the mode amplitude is changed more pronouncedly than in the case when the same inhomogeneities are placed far from them. In the above-mentioned spots the given mode adds in phase with the adjacent modes and therefore energy exchange with the latter is most efficient when scattering takes place at large-scaled inhomogeneities located there. To quantitatively describe the appropriate effects, the notion of Fresnel zones for modes is introduced. Simple and statistical averaging-suited formulae are deduced to calculate mode amplitude variations under scattering at large-scale inhomogeneities which are "mode analogues" of well-known formulae of the Rytov method and of the method of geometrical optics.

[1] Flatte S.M., Dashen R., Munk W., Watson K.M. and Zachariasen F., Sound transmission through a fluctuating ocean (Cambridge U. P., New York, 1979).

[2] Virovlyansky A.L., Kosterin A.G. and Malakhov A.N. Waves in Random Media 1 (1991) 409-418. [3] Felsen L.B. J.Acoust.Soc.Amer. 69 (1981) 352-361. 